\title{
Ophthalmic Examinations Link Group
}

National Cancer Institute

\section{Source}

National Cancer Institute. Ophthalmic Examinations Link Group. NCI Thesaurus. Code C162036.

A sequence of characters used to link multiple ophthalmic examinations to a single finding. 